\title{
Hilfen aus einer Hand
}

\section{Die SGB VIII-Reform aus Sicht der Internationalen Gesellschaft für erzieherische Hilfen}

\begin{abstract}
Nach einem längeren fachlichen Diskussions- und Entwicklungsprozess ist das KJSG am 10.06.2021 in Kraft getreten. Mit ihm ist der sozialpädagogische Grundgedanke des Kinder- und Jugendhilfegesetzes erhalten geblieben und die Forderung nach "Hilfen aus einer Hand" auch im Hinblick auf Leistungen einer inklusiven Kinder- und Jugendhilfe gesetzlich verankert worden. Zudem wurden Beratungs-, Beteiligungs- und Beschwerderechte der Adressat_innen erweitert sowie neue Regelungen zur Erleichterung des Übergangs ins Erwachsenenleben festgelegt. Spannend wird sein, wie sich die aktuelle Umsetzung gestaltet und ob auch die seitens des Fachverbandes geforderten Anliegen erfüllt werden können.
\end{abstract}

$\mathrm{D}$ ie Internationale Gesellschaft für erzieherische Hilfen (IGfH) ist ein bundesweit tätiger Fachverband, der sich als Plattform des sozialpädagogischen Dialogs über erzieherische Hilfen versteht. Vor diesem Hintergrund begleitet der Verband seit mehr als fünf Jahren über zwei Legislaturperioden Versuche, ein „Kinder- und Jugend Stärkungsgesetz - KJSG“ zu verabschieden. Die Geschichte des „neuen“ Gesetzes begann im September 2016 mit einem „Arbeitsent-

\begin{tabular}{|l|l|} 
Nicole Knuth \\
Fachhochschule Dortmund, Dortmund, Deutschland \\
*1973, Studium Lehramt mit den Fächern Englisch und \\
Sozialpädagogik sowie Erziehungswissenschaft, Dr. phil., seit \\
2015 Professorin für Soziale Arbeit mit dem Schwerpunkt Hilfen \\
zur Erziehung an der Fachhochschule Dortmund, Mitglied des \\
Vorstands der IGfH. \\
nicole.knuth@fh-dortmund.de
\end{tabular}

Norbert Struck
Berlin, Deutschland
*1953, Diplom Pädagoge. Von 1991 bis 2018 Jugendhilfereferent
beim Paritätischen Wohlfahrtsverband - Gesamtverband.
Redaktionsmitglied der Fachzeitschrift der IGfH „Forum
Erziehungshilfen"
norbert-struck@t-online.de

Zusammenfassung Der Beitrag gibt eine Gesamteinschätzung zu den Änderungen des SGB VIII durch das KJSG aus der Sicht eines Fachverbandes der erzieherischen Hilfen. Er bilanziert, dass der sozialpädagogische Grundgedanke des Kinder- und Jugendhilfegesetzes erhalten geblieben ist und die Beratungs-, Beteiligungs- und Beschwerderechte der Adressat_innen ausgebaut wurden. Außerdem wurden zentrale Stolpersteine im Übergang ins Erwachsenenleben angegangen und vor allem der Grundgedanke „Hilfen aus einer Hand“ auch im Hinblick auf Leistungen einer inklusiven Kinder- und Jugendhilfe umgesetzt.

Schlüsselwörter Gesetzesreform, Beteiligungs- und Beschwerderechte, Inklusive Kinder- und Jugendhilfe wurf“, der in der Fachwelt empörte Reaktionen auslöste (Struck 2016). Die Modifikationen, die im weiteren Gesetzgebungsprozess der 18. Legislaturperiode vorgenommen wurden, hatten bis zur Verabschiedung des Gesetzes durch den Bundestag Ende Juni 2017 zu einem Gesetz geführt, das aus der Sicht der IGfH einen deutlichen Rückschritt bedeutet hätte. Durch die Nichtbefassung des Bundesrats endete diese Gesetzesinitiative. In der 19. Legislaturperiode wurde das Gesetzesvorhaben wieder auf die Agenda der Koalition gesetzt. Zwischen dem ersten Referatsentwurf vom 05.10.2020 bis zur Verabschiedung des Gesetzes durch den Bundestag am 22.04.2021 hat das Gesetz erneut eine ganze Reihe von Modifikationen erfahren, die sich zunächst im Regierungsentwurf vom 25.01.2021 (BT-Dr. 19/26107), dann in den Empfehlungen des Bundesrates vom 12.02.2021 (BT-Dr. 19/27481), in der Gegenäußerung der Bundesregierung zu diesen Empfehlungen vom 12.03.2021 (BTDr. 19/27481) und schließlich im Änderungsantrag der Koalitionsfraktionen, der am 15.04.2021 im Bundestagsausschuss FSFJ und am 22.04.2021 in 2. und 3. Lesung vom Bundestag beschlossen wurde, niedergeschlagen haben. Der Bundesrat stimmte am 07.05.2021 dem verabschiedeten „Gesetz zur Stärkung von Kindern und Jugendlichen“ (KJSG) zu, so dass dieses am 10.06.2021 in Kraft treten konnte.

Im Hinblick auf diesen Prozess kann man sagen: gut, dass das Gesetzgebungsverfahren in der letzten Legislaturperiode gescheitert ist - gut, dass es in dieser Legislaturperiode verabschiedet wurde - denn zwischen Beginn und Ende liegen fachliche Welten!

Im Folgenden nehmen wir ausgewählte Aspekte der Reform in Bezug auf die Auswirkungen auf die Hilfen zur Erziehung in den Blick und kommentieren sie aus Sicht der IGfH. 


\section{Hilfen aus einer Hand für Kinder und Jugendliche mit und ohne Behinderungen}

Im Gesetzgebungsverfahren war von Anfang an das Ziel einer inklusiven Kinder- und Jugendhilfe vorgegeben. In der 18. Legislaturperiode wurden dazu „Lösungen“ entwickelt, die aus unserer Sicht eindeutig kontraproduktiv waren und sich auf die Verknüpfung von Eingliederungshilfen und Hilfen zur Erziehung fokussiert hatten. Im jetzigen Gesetz ist diese Fixierung zurückgetreten und es werden alle Aufgaben der Kinder- und Jugendhilfe aus der Perspektive Inklusion einbezogen. Der Preis ist ein bis 2028 währender Prozess der Umgestaltung, der in drei Stufen erfolgen soll:

- Erste Stufe: Änderungen zur Gestaltung einer inklusiven Kinder- und Jugendhilfe und Bereinigung der insbesondere zwischen Kinder- und Jugendhilfe und Eingliederungshilfe bestehenden Schnittstellen. Diese Stufe tritt unmittelbar am Tag nach der Verkündung des Gesetzes in Kraft.

- Zweite Stufe: Einführung eines „Verfahrenslotsen“ beim Jugendamt für die Jahre 2024 bis 2028 . Verfahrenslotsen sollen verbindlich Eingliederungshilfekompetenz in die Jugendämter bringen und die Fachlichkeit diesbezüglich gegenüber den Kämmerern stärken. Deshalb ist es sinnvoll, dass diese Verfahrenslotsen beim öffentlichen Träger angesiedelt sind und nicht outgesourct werden können. Kommunen können sie früher installieren - und: sie sollten es auch!

- Dritte Stufe: Übernahme der vorrangigen Zuständigkeit des Trägers der öffentlichen Jugendhilfe für Leistungen der Eingliederungshilfe auch an junge Menschen mit (drohenden) körperlichen oder geistigen Behinderungen im Jahr 2028. Voraussetzung der Realisierung ist allerdings, dass spätestens bis zum 1. Januar 2027 ein Bundesgesetz verkündet worden ist, das diesbezüglich (mindestens) konkrete Regelungen zum leistungsberechtigten Personenkreis, zu Art und Umfang der Leistung und zur Kostenbeteiligung enthält. Zur Vorbereitung werden in $\mathbb{S} 107$ SGB VIII (Übergangsregelungen) detaillierte Untersuchungs- und Berichtsaufträge an das BMFSFJ formuliert.

Auf dem Weg zu einer inklusiven Jugendhilfe gibt es noch einige Unwägbarkeiten und es ist noch völlig unklar, welche finanzpolitischen Fallstricke dem sozialen Bereich in Post-Corona-Zeiten bevorstehen. Aber: Im Rahmen des Art. 1 des KJSG sowie der ergänzenden Regelungen in den weiteren Artikeln des Gesetzes sind Ziele formuliert, Etappen benannt und derzeit keine fachpolitische Fundamentalopposition erkennbar. Daher besteht Grund zu vorsichtigem Optimismus, dass die Hürden überwunden werden und die Kinder- und
Jugendhilfe den aus der Sicht der IGfH notwendigen Inklusionsanspruch inkorporieren und ausgestalten kann und wird.

\section{Rechtsansprüche auf Beratung und Unterstützung für Eltern und Pflegeeltern}

Eltern erhalten - unabhängig von der Wahrnehmung der Personensorge - einen Rechtsanspruch auf Beratung und Unterstützung, wenn ihr Kind in einer Pflegefamilie oder einer Einrichtung der Erziehungshilfe betreut wird. Das Zusammenwirken von Eltern sowie Pflegeoder Erziehungspersonen soll durch eine verbindlichere Unterstützung des Jugendamtes verbessert werden $(\mathbb{S} 37$ SGB VIII). Korrespondierend haben auch die Pflegepersonen einen Rechtsanspruch auf Beratung und Unterstützung ( $\mathbb{3} 37$ a SGB VIII zuvor: $\mathbb{3} 3$ Abs. 2 SGB VIII a. F.). Diese Beratungen können von freien Trägern, die Vereinbarungen nach $\ 77$ Abs. 2 SGB VIII abgeschlossen haben, auf der Basis von Entgelten erbracht werden. Insbesondere der neue Beratungsanspruch für Eltern ist ein wichtiger Meilenstein, um die Zusammenarbeit mit Eltern zu unterstützen und dieser Kontur zu verleihen, so dass dieser - bislang häufig vernachlässigte - Bereich hoffentlich mehr Aufmerksamkeit erfährt. Bei der Umsetzung muss jedoch dringend darauf geachtet werden, dass der neue Rechtsanspruch nicht zu einer weiteren Versäulung von Hilfen führt. So wird es darum gehen, eigenständige Unterstützungs- und Beratungsangebote aufzubauen, gleichzeitig aber auch Beratungs- und Unterstützungsleistungen für Eltern zu fördern, die eng verknüpft sind mit den Hilfsangeboten für ihre Kinder und z. B. durch Fachkräfte der Heimeinrichtungen oder der Pflegekinderdienste angeboten werden.

Um Verunsicherungen des Kindes oder Jugendlichen zu reduzieren und Transparenz und Kontinuität herzustellen, wird u. a. eine prozesshafte Perspektivklärung als Bestandteil der Hilfeplanung bei vollstationären Hilfen explizit geregelt ( $\$ 37$ c SGB VIII). Im Rahmen der Hilfeplanung ist künftig auch Geschwisterbeziehungen Rechnung zu tragen ( $\$ 36$ SGB VIII).

Aus Sicht der IGfH ist es sehr zu unterstützen, dass in der Hilfeplanung nun explizit ein Fokus auf die Perspektivklärung gelegt wird und diese gemeinsam mit den Betroffenen prozesshaft gestaltet werden muss. Inwieweit die Hilfeplanung aber tatsächlich auf Dauer angelegte Lebensperspektiven erarbeiten kann, erscheint fraglich, denn auch in Pflegefamilien sind Entwicklungen der Erziehungssituationen nicht unbedingt absehbar.

In $\ 1632$ Absatz 4 BGB wird bestimmt, dass das Familiengericht von Amts wegen oder auf Antrag der Pflegeperson anordnen kann, dass der Verbleib eines Pflegekindes bei der Pflegeperson auf Dauer ist. Eine solche 
Anordnung ist allerdings auf Antrag der Eltern aufzuheben, wenn die Wegnahme des Kindes von der Pflegeperson das Kindeswohl nicht gefährdet ( $\mathbb{S} 1696$ Abs. 3 BGB).

\section{Das Regelwerk der ambulanten Hilfen zur Erziehung wird ausgeweitet}

Die Möglichkeiten der direkten Inanspruchnahme einer Unterstützung werden explizit um Hilfen für Familien in Notsituationen $(\mathbb{S} 20$ SGB VIII) erweitert $(\mathbb{S} 36 \mathrm{a}$ Abs. 2 SGB VIII). Schlussendlich wurde verhindert, dass der Regelungsgehalt des $\$ 20$ SGB VIII in den Abschnitt der Hilfen zur Erziehung als $\mathbb{2} 28$ a SGB VIII überführt wurde. Allerdings ist es aufgrund dieser Fokussierung auf eine Verortung in den Hilfen zur Erziehung nicht mehr gelungen, den $\mathbb{2 0}$ SGB VIII fachlich weiterzuentwickeln. Im Hinblick auf die Altersbeschränkungen ( „Kinder“) und z. B. auf die Konkretisierung von Hilfen für Eltern mit Behinderungen wären hier viele sinnvolle Weiterentwicklungen möglich gewesen, wenn nicht die völlig überflüssige Intention, die Leistung in den Hilfen zur Erziehung zu verankern, alles überlagert hätte.

Der $\mathbb{S} 77$ SGB VIII wird gänzlich neugestaltet und heißt nun: „Vereinbarungen über Kostenübernahme und Qualitätsentwicklung bei ambulanten Leistungen “. Diese Neufassung wird sicherlich noch einige rechtsdogmatische Nachwehen haben, weil dadurch ein systematischer Ort fehlt, durch den Infrastruktur z. B. der Jugendarbeit oder Familienarbeit auch durch zweiseitige Leistungsverträge abgesichert werden konnte, anstatt nur über den Weg der Förderung nach $\$ 74$ SGB VIII. Jedenfalls sollen künftig bei ambulanten Leistungen Vereinbarungen zu Inhalt, Umfang, Qualität, Grundsätzen und Kriterien zur Bewertung von Qualität und Maßnahmen zu ihrer Gewährleistung zwischen dem Leistungserbringer und dem Jugendamt abgeschlossen werden. Merkwürdigerweise fehlt dabei die Erwähnung der Entgelte, die sich daraus ergeben. Ob „das Nähere“, das das Landesrecht dazu regeln soll, die Lücke füllen wird, ist ungewiss.

Im Kostenrecht haben wir somit die Förderung ( $\mathbb{S} 74)$, die Kostenübernahme bei ambulanten Leistungen ( $\mathbb{S 7 7}$ ) und die Entgeltregelungen bei (teil)stationären Leistungen in den $\mathbb{S}$ 78a-78g SGB VIII - und den „Wegduckparagrafen“ 74a SGB VIII, der den Ländern die KitaFinanzierung überlässt.

Stärkung von Kindern und Jugendlichen in Pflegefamilien und Einrichtungen der Erziehungshilfe

Die Kostenbeteiligung von jungen Menschen bei vollstationären Leistungen wird auf höchstens $25 \%$ ihres Einkommens reduziert. Einkommen aus Praktika, Feri- enjobs, ehrenamtlichen Tätigkeiten und $150 €$ der Ausbildungsvergütung bleiben dabei unberücksichtigt ( $\$ 94$ Abs. 6). Allerdings wird nun das aktuelle Einkommen zugrunde gelegt und nicht mehr das des Vorjahres. ${ }^{1}$ Von der Kostenheranziehung junger Volljähriger aus dem Vermögen wird gänzlich abgesehen.

Die Rechtsposition junger Volljähriger wird in $\mathbb{4} 41$ gestärkt. Careleaver werden bei ihren Schritten in ein selbständiges Erwachsenenleben verbindlicher begleitet und unterstützt. Ihnen wird eine Rückkehroption eingeräumt $(\mathbb{S} 41$ Abs. 1$)$. Mit $\mathbb{4} 41$ a wird die „Nachbetreuung “ festgeschrieben und in $\$ 36$ b wird die „Zuständigkeit beim Übergang“ in andere Sozialleistungssysteme geregelt, die „,in der Regel ein Jahr vor dem voraussichtlichen Zuständigkeitswechsel“ zu beginnen hat.

In diesen Teilen hat sich das Engagement des Careleaver e. V. und seiner Netzwerke deutlich positiv niedergeschlagen, was auch in der Entschließung des Deutschen Bundestages (s. BT-Dr. 19/28870, S. 11) deutlich anerkannt wird. Kein „Wünsch Dir was“, aber doch deutliche Fortschritte, die von der IGfH mit Nachdruck unterstützt werden, auch wenn der Fachverband zum Beispiel deutlich die vollständige Abschaffung der Kostenbeteiligung gefordert hatte.

Zur Stärkung der Bedarfsgerechtigkeit von Hilfen wird zudem klargestellt, dass unterschiedliche erzieherische Hilfen miteinander kombiniert werden können. Das ist eine unproblematische Klarstellung, die aber in unterschiedlichen Bereichen von zentraler Bedeutung ist (z. B. für Pflegefamilien, um zusätzliche Hilfsangebote zu erhalten oder auch für Eltern, deren Kinder in Heimerziehung leben).

Warum die für Eingliederungshilfen ( $\$ 35$ a und künftig auch andere junge Menschen mit Behinderungen) sinnvolle Bestimmung, dass ausbildungsbezogene Leistungen auch als Gruppenangebote gestaltet werden können, in $\$ 27$ Abs. 3 bei den Hilfen zur Erziehung verortet wurde, ist allerdings nicht nachvollziehbar und auch die Gesetzesbegründungen geben dafür nichts her: ein Strickfehler.

\section{Mehr Beteiligung von jungen Menschen, Eltern und Familien}

In diesem Punkt finden viele Forderungen und Entwicklungen der Fachdiskurse und des fachlichen Engagements erfreulichen Niederschlag. Das betrifft die Einrichtung von Ombudsstellen $(\mathbb{S} 9 \mathrm{a})$ - der noch jungen, aber sehr wirkungsvollen Ombudsstellen-Bewegung angemessen zunächst nur als Verpflichtung der Länder zur Schaffung einer bedarfsgerechten Infrastruktur. Jetzt kommt es darauf an, die Ombudsstellenarbeit in den Landesausführungsgesetzen klug zu verankern und 
dabei Unabhängigkeit, Weisungsungebundenheit und Fachlichkeit abzusichern. Es wird ein Lernprozess sein, wie in den Flächenländern die kommunalen Aktivitäten und die Initiativen von Landesebene aus so aufeinander bezogen werden, dass keine „Fakes“ entstehen, sondern eine bedarfsgerechte Struktur von Ombudsstellen, die ihre Intention, die Machtasymmetrie von Betroffenen, Leistungsberechtigten und Leistungsgewährern und -erbringern zu reduzieren, verwirklichen. Das Bundesnetzwerk Ombudschaft (https://ombudschaft-jugendhilfe. de/) sollte dabei aus unserer Sicht ein_e wichtige Moderator_in sein.

Die Stärkung von Selbstvertretung und Selbsthilfe $(\mathbb{S} 4 \mathrm{a})$ wird auf kommunaler und überörtlicher Ebene verankert („die öffentliche Jugendhilfe“). Die stärkeren Impulse zur Selbstvertretung sind bisher eher auf Landes- und auch Bundesebene verortet (wie zum Beispiel Momo; Careleaver e. V.; Jugendliche ohne Grenzen). Abzuwarten bleibt, ob in den kommunalen Strukturen Zeit und Geld investiert wird, um den Aufbau tragfähiger neuer Strukturen zu unterstützen und so auch neue Netzwerke aufzubauen. Das Zukunftsforum Heimerziehung (2021) hat gezeigt, dass zum Beispiel Eltern sehr an einem Aufbau von Selbsthilfeorganisationen interessiert, aber aufgrund ihrer Situation auf eine Unterstützung bei der Vernetzung angewiesen sind.

Das Gesetz fordert schließlich die verbindliche Implementierung auch von externen Beschwerdemöglichkeiten für junge Menschen in Einrichtungen ( $\mathbb{S} 45$ Abs. 2 Nr. 4) und für Pflegekinder ( $\ 37 \mathrm{~b})$. Das ist gut so. Und ebenso ist es richtig, dass der Beratungsanspruch für Kinder und Jugendliche nach $\mathbb{S} 8$ Abs. 3 nicht mehr durch das Erfordernis einer „Not- und Konfliktlage“ eingeschränkt wird und eine Pflicht zur „verständlichen und umfassenden Aufklärung, Beratung “ und Beteiligung in unterschiedlichen Aufgabenkontexten konstituiert wird.

\section{Betriebserlaubnisverfahren und Zulässigkeit von Auslandsmaßnahmen}

Das Betriebserlaubnisverfahren wurde neugestaltet. In $\mathbb{4} 45$ a gibt es jetzt eine Legaldefinition des Einrichtungsbegriffs. Es wird die „Zuverlässigkeit“ des Trägers gefordert ( $\$ 45$ Abs. 2) und der Anknüpfungspunkt wird von der individuellen Kindeswohlgefährdung auf eine strukturelle Sicherung des Kindeswohls verschoben - eine überfällige Klarstellung. Die Bestimmungen zur Zulässigkeit von Auslandsmaßnahmen werden jetzt in $\mathbb{3} 38$ gebündelt. Zentral ist, dass der örtliche Träger sich vor Ort von der Struktur und Qualität der Leistung informieren muss und Hilfeplangespräche vor Ort führen soll. Auslandsmaßnahmen können nur von Trä- gern durchgeführt werden, die in Deutschland eine betriebserlaubnispflichtige Einrichtung haben. Die deutliche Verpflichtung zur Hilfeplanung und Überprüfung am Ort der Leistungserbringung stellen aus Sicht der IGfH notwendige und fachlich dringend erforderliche Anforderungen dar, um die Rechte und das Wohl junger Menschen bei Auslandsmaßnahmen besser als bisher zu gewährleisten.

\section{Fazit}

Mit Blick auf das Anliegen der IGfH ist hinsichtlich der Einschätzung des KJSG insbesondere festzuhalten, dass der sozialpädagogische Grundgedanke des Kinder- und Jugendhilfegesetzes erhalten geblieben ist und die Beratungs-, Beteiligungs- und Beschwerderechte der Adressat_innen ausgebaut wurden. Außerdem wurden zentrale Stolpersteine im Übergang ins Erwachsenenleben angegangen und vor allem der Grundgedanke „Hilfen aus einer Hand “ auch im Hinblick auf Leistungen einer inklusiven Kinder- und Jugendhilfe umgesetzt.

In den nächsten Jahren wird sich zeigen, inwieweit dieser inklusive Gedanke tatsächlich in praxistauglichen Konzepten ausgearbeitet und in Strukturen mit verlässlichen Ansprechpartner_innen für Hilfesuchende verankert wird. Ähnliches gilt auch für die verbesserten Beratungs- und Beteiligungsrechte. Die rechtlichen Vorgaben ermöglichen neue Wege - es bleibt abzuwarten, ob es an der einen oder anderen Stelle nicht noch Nachbesserungen bedarf, um die tatsächlich dringend notwendige Umsetzung voran zu treiben.

Eingegangen. 2. Juni 2021

Angenommen. 15. September 2021

Funding. Open Access funding enabled and organized by Projekt DEAL.

Open Access. Dieser Artikel wird unter der Creative Commons Namensnennung 4.0 International Lizenz veröffentlicht, welche die Nutzung, Vervielfältigung, Bearbeitung, Verbreitung und Wiedergabe in jeglichem Medium und Format erlaubt, sofern Sie den/die ursprünglichen Autor(en) und die Quelle ordnungsgemäß nennen, einen Link zur Creative Commons Lizenz beifügen und angeben, ob Änderungen vorgenommen wurden.

Die in diesem Artikel enthaltenen Bilder und sonstiges Drittmaterial unterliegen ebenfalls der genannten Creative Commons Lizenz, sofern sich aus der Abbildungslegende nichts anderes ergibt. Sofern das betreffende Material nicht unter der genannten Creative Commons Lizenz steht und die betreffende Handlung nicht nach gesetzlichen Vorschriften erlaubt ist, ist für die oben aufgeführten Weiterverwendungen des Materials die Einwilligung des jeweiligen Rechteinhabers einzuholen.

Weitere Details zur Lizenz entnehmen Sie bitte der Lizenzinformation auf http://creativecommons.org/licenses/by/4.0/deed.de. 
1. Bis zum Inkrafttreten des KJSG haben viele Jugendämter - entsprechend einer Empfehlung der BAG LJÄ-rechtswidrig die bisher geltende Rechtslage zuungunsten der jungen Menschen nicht angewandt. Das Bundesverwaltungsgericht hat mit seinem Urteil vom 11.12.2020 - BVerwG 5C 9.19ECLI abschließend diesen Rechtsbruch markiert.

\section{Literatur}

Struck, N. (2016). Kommt das „neue SGB VIII - Leistungserbringung für Kinder, Jugendliche und Familien"? Forum Erziehungshilfen, 22(5), 260264.

Zukunftsforum Heimerziehung (2021). Zukunftsimpulse für die „Heimerziehung". Eine nachhaltige Infrastruktur mit jungen Menschen gestalten! Frankfurt/M.: IGfH-Eigenverlag.

Hier steht eine Anzeige.

黛 Springer 